\title{
Flechtenkartierung als Grundlage für die Charakterisierung der Luftbelastung (Bündner Rheintal)
}

\section{Einleitung}

Es ist üblich, Luftverschmutzung mit Hilfe von technischen Meßgeräten zu erfassen und zu charakterisieren. Wo die Art der Luftverunreinigung unbekannt, der Luftschadstoff mit den herkömmlichen Meßgeräten nicht erfaßbar ist, oder wo Auswirkungen und Folgen der Luftverschmutzung abgeschätzt werden sollen, sind die technischen Verfahren unzulänglich. Bioindikationsmethoden können diese Informationslücken schließen.

Ideale Bioindikatoren sind Flechten; sie verfügen über eine lange Tradition als Zeigerorganismen der Luftverschmutzung (KIRSCHBAUM 1986; LEBLANC. DESLOOVER 1970; MACHER 1987, NASH 1976).

Im Rahmen des Nationalen Forschungsprogrammes 14 "Luftverschmutzung und Lufthaushalt in der Schweiz» wurde unter der Leitung von Dr. Klaus Ammann eine praxisorientierte, einfache und quantitative Bioindikations-Methode entwickelt (HERZIG, LIEBENDÖRFER, URECH 1985; URECH. LIEBENDÖRFER. HERZIG 1986). Diese von der «Arbeitsgemeinschaft Bioindikation» erarbeitete «kalibrierte Flechtenmethode zur Luftgütebewertung» erlaubt die flächendeckende sowie punktuelle Erfassung der lufthygienischen Gesamtbelastung im Schweizer Mittelland.

Die Fallstudie «Ökologische Planung Bündner Rheintal» des ORL-Projektes «Grundlagen und Möglichkeiten ökologischer Planung» (FLEPP. SCHMID, TRACHSLER 1984; GFELLER, KIAS. SCHILTER. SCHMID. TRACHSLER, PETER. THOENI 1987; TRACHSLER. KIAS 1986) sucht Verfahren, welche geeignet sind, mit kleinem Aufwand Informationen über die natürlichen Lebensgrundlagen zu liefern und diese für die Raumplanung bereitzustellen. Im Aussagebereich Luft liegt die Anwendung der Bioindikation mit Flechten aus diesen Gründen auf der Hand. Diese Arbeiten wurden unter der Leitung von Dr. Klaus Ammann und mit der Beratung von Rolf Herzig, Luzius Liebendörfer und Martin Urech durchgeführt. Die Mitarbeiter des ORL stellten sich oft hilfsbereit zur Seite. Das Kantonale Amt für Umweltschutz des Kantons Graubünden begrüßte und unterstützte das vorliegende Projekt in verdankenswerter Weise.

\section{Voraussetzungen}

Die «kalibrierte Flechtenindikationsmethode» ist in ihrer Anwendung auf Gebiete mit einem für das Schweizer Mittelland vergleichbaren Flechtenartenspektrum begrenzt. Erste floristische Untersuchungen wurden in Zusammenarbeit mit Sibylle Grundlehner zur Abklärung der Vergleichbarkeit durchgeführt. Die floristische Beschreibung des Bündner Rheintales ergab, da $B$ die Artenzusammensetzung der aufgrund der klimatischen Bedingungen vorherrschenden Flechtenflora sich von derjenigen des Schweizer Mittellandes unterscheidet. Die «kalibrierte Flechtenindikationsmethode» kann übernommen, doch muß sie an die Verhältnisse des Bündner Rheintales angepaßt werden. Es drängte sich die Bearbeitung folgender methodischer Problemstellungen auf:

- Es muß die Artenkombination gefunden werden, welche die immissionsökologische Situation optimal wiedergibt.

- Die immissionsrelevante Aussage mittels Flechten muß bestätigt und die Güte der Übereinstimmung zwischen Flechten- und Immissionsdaten für das Bündner Rheintal ermittelt werden.

- Die gefundenen Flechtenindices werden neu klassiert und die Flechtenzonen bezüglich Wirkung und Gesamtbelastung beschrieben.

\section{Methode}

Die Bioindikationsmethode mit Flechten erfaßt die epiphytische Flechtenflora an freistehenden Bäumen in Städten und in ländlichen, waldfreien Gebieten bis auf $1000 \mathrm{~m}$ Meereshöhe. Die Trägerbäume werden nach bestimmten, streng standardisierten Kriterien ausgewählt (HERZIG. LIEBENDÖRFER. URECH 1987). Für das Bündner Rheintal wurde der Walnußbaum (Juglans regia) zusätzlich als Trägerbaum evaluiert.

Kathrin Peter, dipl. Biologin, Arbeitsgemeinschaft Bioindikation, Systematisch-Geobotanisches Institut, Altenbergrain 21,3013 Bern 
An diesen Trägerbäumen werden die Flechtendaten gemäß dem Frequenzverfahren der «kalibrierten Flechten-Indikationsmethode» erhoben. Die Flechten werden innerhalb eines speziell entwickelten zehnfeldrigen Frequenzgitters erfaßt, welches auf einer mittleren Höhe von $145 \mathrm{~cm}$ den halben Stammumfang umfaßt. Die Frequenz bezeichnet die Anzahl Teilfelder des Frequenzgitters, in welchen eine bestimmte Flechtenart auftritt. Die Summe der Frequenzwerte aller gezählten Flechtenarten und -gruppen ergibt den $\operatorname{IAP}_{\mathrm{BR}}$ total pro Baum (Index of Atmospheric Purity/Luftgütewert). Der Luftgütewert ist hoch, wenn viele Flechten vorhanden sind; er ist entsprechend tiefer, wenn die Luft belasteter ist, also wenig Flechten angetroffen werden können.

Eine begleitende Immissionsmeßkampagne wurde zur Kalibrierung der «Bündner Rheintaler Flechtenmethode» durchgeführt. Die zehn Meßstationen wurden so über das Bündner Rheintal verteilt, daß sie möglichst das ganze Spektrum der potentiellen Luftbelastung erfassen. Mit Absorptions- und Depositionsgeräten (Liesegang, Bergerhoff, Regensammler, $\mathrm{NO}_{2}$-Passivsammler) wurden an diesen Standorten neun wichtige Luftschadstoffe $(\mathrm{HCl} /$ $\mathrm{NO}_{2} / \mathrm{NH}_{4} / \mathrm{SO}_{2} / \mathrm{Cd} / \mathrm{Cu} / \mathrm{Pb} / \mathrm{Zn} /$ Staubniederschlag) über ein Jahr gemessen. Die Immissionsdaten lagen in Form von Jahresmittelwerten pro Meßstation vor. Im Umkreis von $250 \mathrm{~m}$ um eine Meßstation herum wurden zehn Bäume auf ihre Flechtenvegetation hin untersucht. Die Flechtendaten lagen in Form von Meßstations-Mittelwerten vor.

\section{Artenauswahl}

Der IAP $_{\mathrm{BR}}$ total setzt sich aus insgesamt 54 Flechtenarten und -gruppen zusammen. Darunter befinden sich solche Arten, deren Verhalten und Verbreitung nicht primär immissions-ökologisch bedingt sind. Diese Flechten dürfen, da sie die Aussage der Luftgüte verwischen, beim IAP $P_{B R}$ nicht berücksichtigt werden. Zum Artenausschluß dienten Verbreitungskarten, Toxitoleranzwerte $(\mathrm{Ma}$ für die Sensibilität jeder Flechtenart gegenüber der Luftverschmutzung) und mittlerer Frequenzanteil als Entscheidungshilfen.

Das Verhalten der berücksichtigten Arten sollte möglichst demjenigen von Parmelia subargentifera (Abb. 1) entsprechen: mit steigendem IAP ${ }_{\mathrm{BR}}$ total steigt auch der mittlere Frequenzanteil.

Physcia orbicularis-Gruppe (Abb. 2) ist Beispiel für eine indifferente Flechtenartengruppe. Ihr mittlerer Frequenzanteil ist, unabhängig von der Höhe des $\mathrm{IAP}_{\mathrm{BR}}$ total, an allen Meßstationen ungefähr gleich hoch.

Physcia nigricans (Abb. 3) ist Beispiel einer dem IAP-Konzept gegenläufigen Art. Ihr mittlerer Frequenzanteil sinkt mit zunehmendem Luftgütewert.
Diese zwei letzten Arten wurden ausgeschlossen. Der IAP $_{B R}$ setzt sich aus insgesamt 51 Flechtenarten und -gruppen zusammen. Nur drei Arten wurden ausgeschlossen. Sie zeigen gleichzeitig auch die tiefsten aller Toxitoleranzwerte.

Parmelia subargentifera

Mittlerer Frequenzonteil an den Immissionstationen

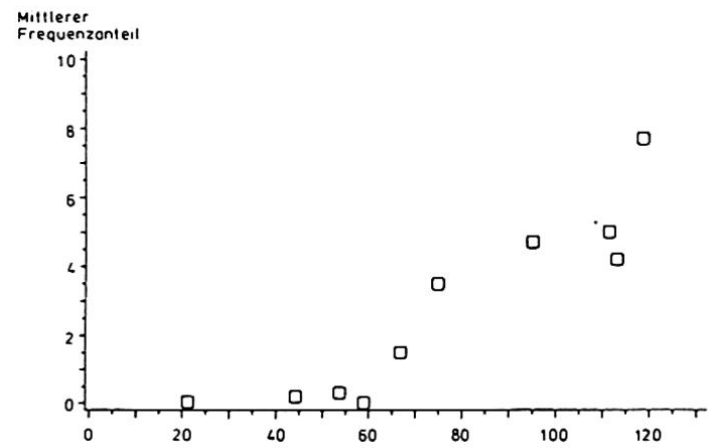

Abb. 1 Parmelia subargentifera: ${ }_{\text {IAP }}^{\text {Ohn totol }}$ ohne
Parmelio
surorgention immissionsökologisch relevantes Verhalten

Physcia orbicularis - Gruppe Mittlerer Frequenzonteil on den immissionstotionen

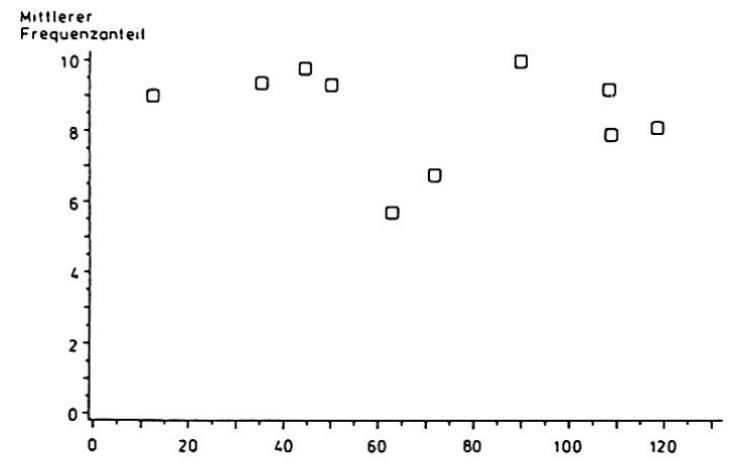

Abb. 2 Physcia orbicularis-Gruppe: IAP
one told
Physcio
obricularis immissionsökologisch indifferentes Verhalten

Physcio nigricans

Milllerer Frequenzontell on den Immissionstotionen

Mittlerer

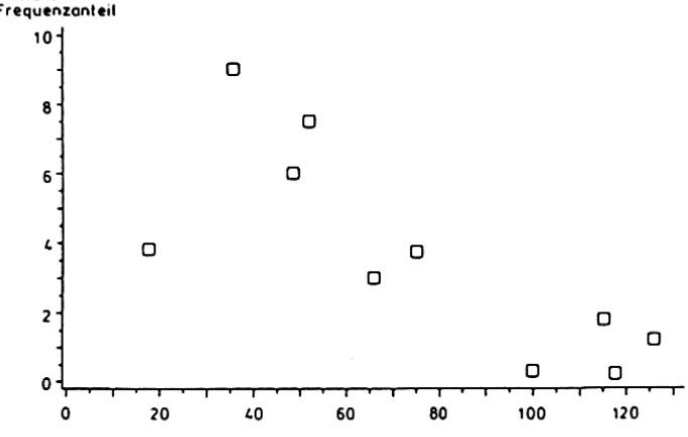

Abb. 3 Physcia nigricans: dem IAP-Konzept gegenläufige Art 


\section{Kalibrierung}

Mit Hilfe der multiplen linearen Regressionsanalyse werden die Flechtendaten mit den technischen Immissionsdaten verglichen. Die multiple lineare Regression zeigt, wie groß die Übereinstimmung zwischen den beiden Datensätzen ist. Zehn Meßstationen bilden einen zu kleinen Stichprobenumfang, um mit einem Neuner-Schadstoffkollektiv rechnen zu können. Daher wurden nur Modelle mit maximal vier Schadstoffvariablen dem multivariaten statistischen Vergleich unterzogen. Auch diese Modelle sollten nur mit der nötigen Vorsicht interpretiert werden.

Das Regressionsmodell mit dem Schadstoffkollektiv $\mathrm{NO}_{2}, \mathrm{SO}_{2}$, Staubniederschlag und $\mathrm{Pb}$ (Abb. 4) weist eine hohe statistische Güte mit dem Bestimmtheitsmaß $\mathrm{R}^{2}=0,94$ und einer deskriptiven Sicherheitsschwelle weit unter der Fünfprozentgrenze auf. Das ausgezeichnete Bestimmtheitsmaß bedeutet, daß $94 \%$ der Variabilität, oder $94 \%$ der Abweichungen der Flechtenflora, an den Meßstationen durch die unterschiedliche Belastung der vier Schadvariablen erklärbar ist. Der Graph der Residuenanalyse (Abb. 4) zeigt die multiple Korrelation zwischen den im Gelände gemessenen IAP $_{\mathrm{BR}}$-Werten und den im Regressionsmodell aus dem Schadstoffkollektiv berechneten Luftgütewerten.

REGRESSIONSMODELL

Bündner Rheintal

Modell mit $\mathrm{SO}_{2}, \mathrm{NO}_{2}$, Staubniederschlag. $\mathrm{Pb}$ an 10 Stationen

$\mathrm{R}^{2}=0,94, \quad a=0,25 \%$

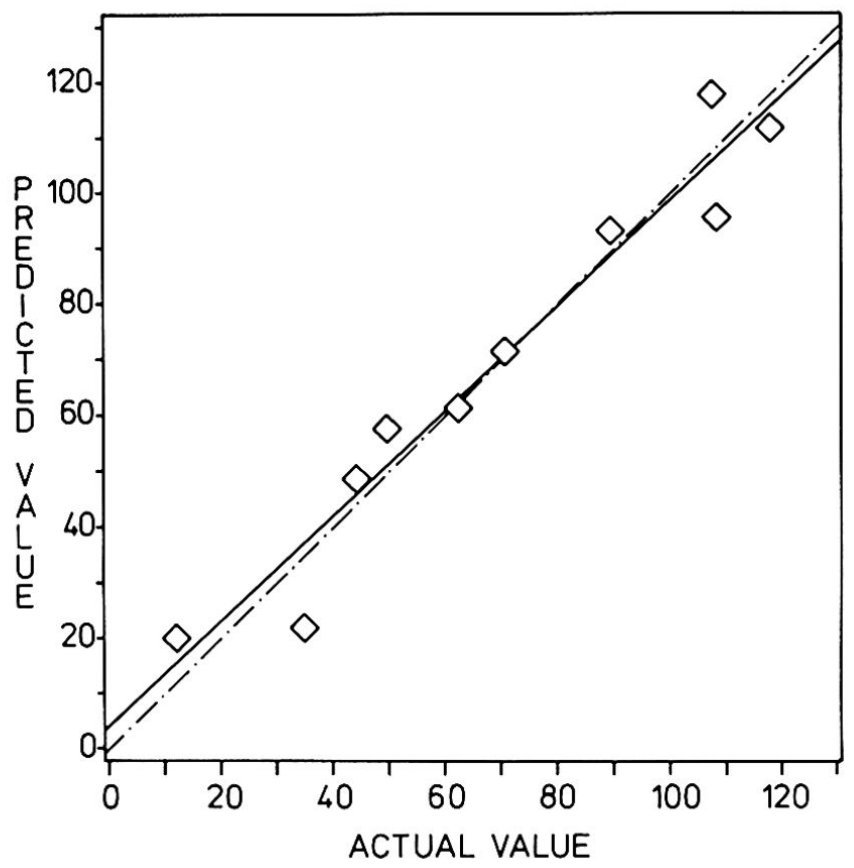

Abb. 4 Regressionsmodell mit vier Schadstoffvariablen
Regressionsrechnungen mit anderen Vierer- und Dreierschadstoffkollektiven ergeben ebensogute Bestimmtheitsmaße.

Dank der Kalibrierung der Flechten- mit den Immissionsdaten können folgende Schlüsse gezogen werden:

- Der IAP $P_{B R}$ gibt einen gesicherten Rückschluß auf die Gesamtbelastung der Luft.

- Die Flechtenzonen dürfen als Luftgesamtbelastungszonen interpretiert werden.

\section{Ergebnisse}

Im Sommer 1986 wurde unter der Mitarbeit von Dr. Marilen Macher von 704 Bäumen die Flechtenflora kartiert. Benachbarte Trägerbäume unter vergleichbaren geographischen Bedingungen wurden zu Georäumen zusammengezogen. Für jeden Georaum wurden der Schwerpunkt der Baumstandorte und der $\mathrm{IAP}_{\mathrm{BR}}$-Mittelwert berechnet.

Die IAP $_{\mathrm{BR}}$-Georaummittelwerte sowie die Schwerpunkte bilden die Grundlage zur Herstellung der Flechtenkarte, die mittels linearer Interpolation konstruiert wird.

Die $\mathrm{IAP}_{\mathrm{BR}^{-}}$-Georaummittelwerte verteilen sich annähernd normal und können mit Hilfe von Mittelwert und Standardabweichung (Abb.6) in fünf mehr oder weniger lineare Zonen eingeteilt werden. Diese Zonen gelten nur für das Bündner Rheintal und angrenzende Gebiete und sind mit den Schweizer Mittellandzonen von HERZIG. LIEBENDÖRFER, URECH 1987 weder identisch noch vergleichbar.

Die Zone mit «sehr wenig Flechtenbewuchs» ist charakterisiert durch eine durchschnittliche Artenzahl pro Baum von 5.4 Arten. Von den 54 Arten und Gruppen sind in dieser Zone nur noch 17 unempfindliche anzutreffen. Hier haben die für das $\mathrm{IAP}_{\mathrm{BR}}-$ Konzept ausgeschlossenen drei Arten ihre stärkste Verbreitung. Diese Zone weist eine "große Gesamt-Immissionsbelastung» auf.

Die Zone mit «wenig Flechtenbewuchs» entspricht einer «relativ großen Gesamtbelastung». Die durchschnittliche Artenzahl ist auf 10.2 gestiegen. In dieser Zone sind 28 Arten angetroffen worden.

Die durchschnittliche Artenzahl pro Baum und die Anzahl der gefundenen Arten steigt mit sinkender Gesamt-Luftbelastung, bis sie in der Zone mit «normalem Flechtenbewuchs" und "sehr kleiner Gesamtbelastung» Werte von 20.4 Arten pro Baum und von insgesamt 36 meist empfindlichen Arten annimmt.

Das Resultat der Flechtenkartierung liegt in Form einer fünfstufigen Luftgütekarte (Abb. 5) vor.

Diese Karte zeigt, wo die lufthygienisch relevanten Problemgebiete der Immissionen liegen. Stadtzentrum Chur, stark belastete Dorfkerne, der relativ hoch belastete Raum um die Kehrichtverbren- 
OEKOLOGISCHE PLANUNG BUENDNER RHEINTAL

BIOINDIKATION MIT FLECHTEN GESAMTBELASTUNG LUFT

WIRKUNGSKATASTER:

SEHR WENIG FLECHTENBEWUCHS

WENIG FLECHTENBEWUCHS

| InI MITTLERER FLECHTENBEWUCHS

册 VIEL FLECHTENBEWUCHS

|II NORMALER FLECHTENBEWUCHS

IMMISSIONSKATASTER:

GROSSE GESAMTBELASTUNG

RELATIV GROSSE GESAMTBELASTUNG

InIm MAESSIGE GESAMTBELASTUNG

KLEINE GESAMTBELASTUNG

III SEHR KLEINE GESAMTBELASTUNG
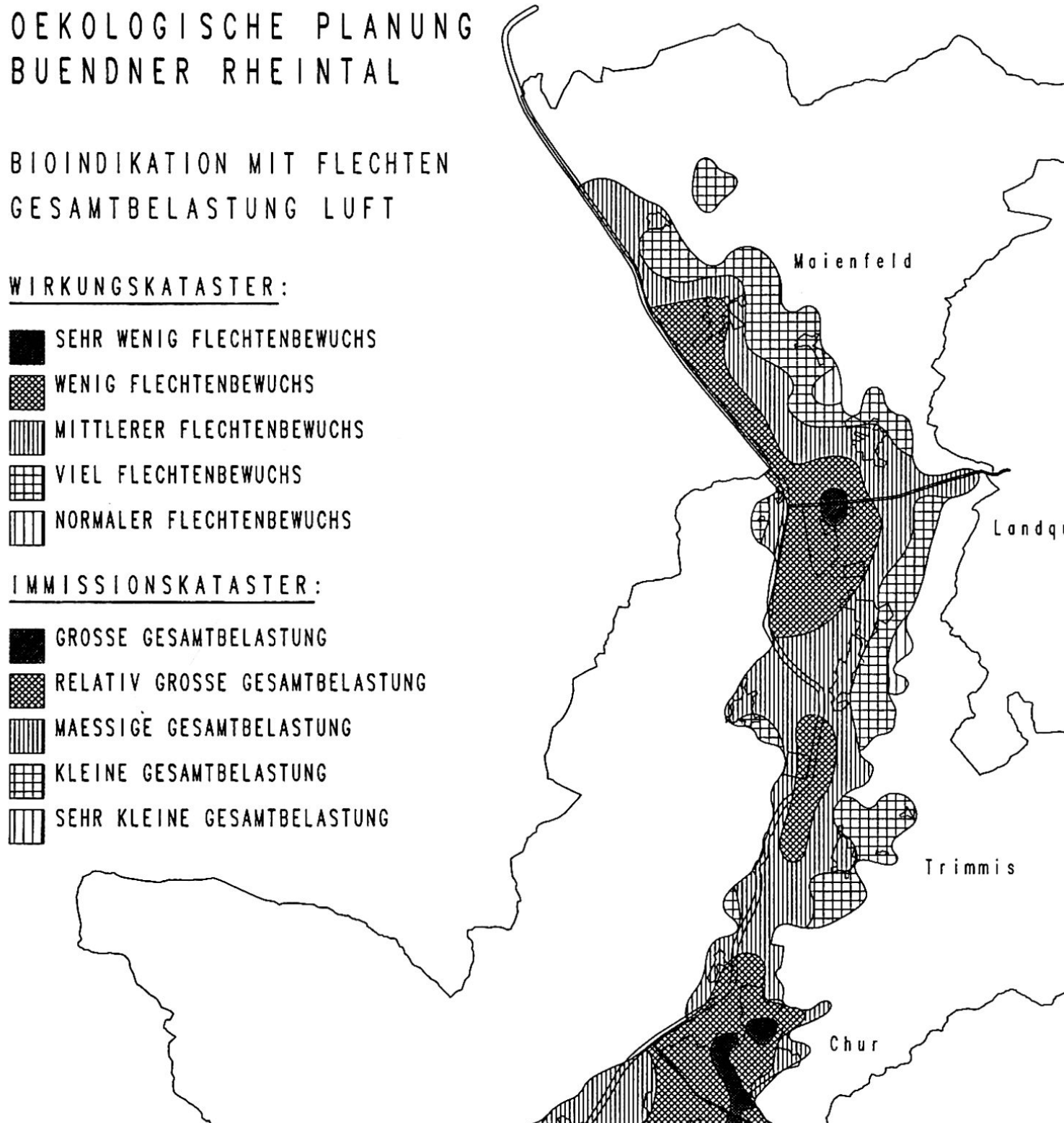


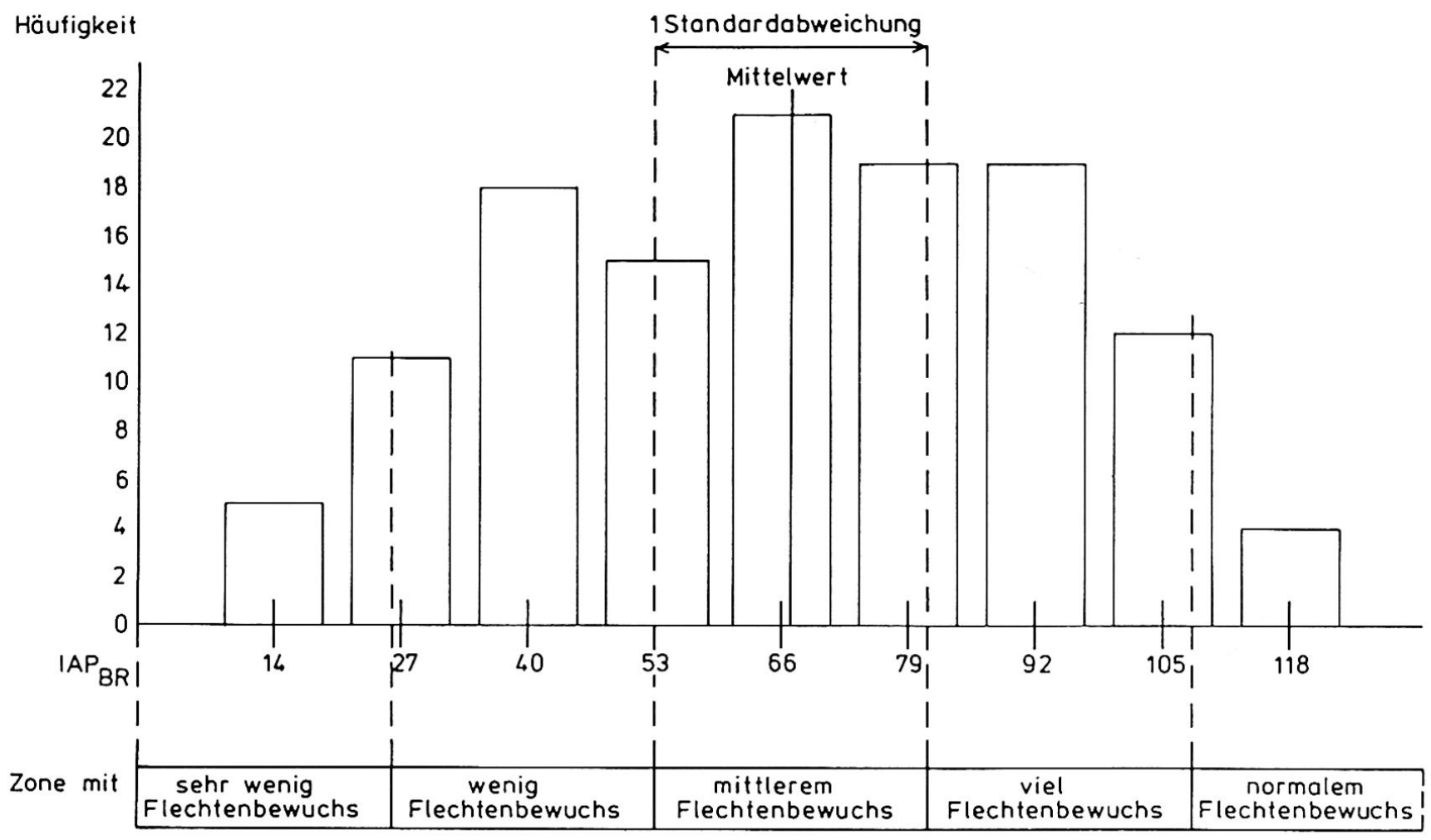

Abb. 6 Verteilung der $\mathrm{IAP}_{\mathrm{BR}}$-Georaummittelwerte

nungsanlage, industrienahe Gebiete sowie stark frequentierte Hauptverkehrsachsen treten deutlich als belastete Zonen hervor. Diese Gebiete sollten bei raumplanerischen Entscheiden und bei Massnahmenplänen besondere Beachtung finden.

Die Karte scheidet auch diejenigen Gebiete aus, die über eine «kleine oder sehr kleine lufthygienische Gesamtbelastung» verfügen.

Die Bioindikationsmethode mit Flechten erlaubt, technische Luft-Meßstationen bezüglich der Gesamtbelastung zu definieren, sie einer entsprechenden Immissionszone zuzuordnen (Abb. 7) und sie untereinander zu vergleichen.

Es bestehen Absichten, die «Bündner Rheintaler Flechtenmethode» mit zusätzlichen technischen Meßdaten, insbesondere Konzentrationsdaten, weiter abzustützen.

\section{Bedeutung der Bioindikation mit Flechten}

Bioindikation ersetzt weder technische Messungen, noch können technische Meßverfahren die gleichen Aussagen wie Bioindikationsmethoden liefern. Die beiden Verfahren ergänzen sich, wenn es darum geht, Ausmaß und Art der Luftverschmutzung sowie Wirkung und Folgen der Immissionsbelastung abzuschätzen.

Bioindikatoren sind lebende Meßinstrumente und so repräsentativ für andere Lebewesen. Sie registrieren integrativ alle wirkungsrelevanten Schadstoffe und ihre synergetisch und antagonistisch wirkenden Gemische. Flechten sind zudem anschauliches Umwelterziehungsmaterial: ihre Reaktion auf die Luftverschmutzung ist sinnlich wahrnehmbar.

Technische Messungen sind da notwendig, wo es darum geht, Konzentrationen und Depositionen einzelner Schadstoffe sowie momentane Spitzenbelastungen $\mathrm{zu}$ erfassen. Genaue quantitative und zeitliche Überwachung einzelner Luftschadstoffe ist nur mit technischen Messungen möglich.

Die Bioindikationsmethode mit Flechten mißt die Gesamt-Immissionsbelastung der Luft. Der Einsatz von Bioindikatoren ist kostengünstig. Er ermöglicht den Aufbau eines engmaschigen Meßnetzes und damit neben der punktuellen auch die fein räumlich differenzierte Erfassung der Gesamtbelastung. Die Anwendung der Flechtenmethode gibt einen raschen und detaillierten Überblick über das Gesamt-Immissionsmuster eines Raumes. 


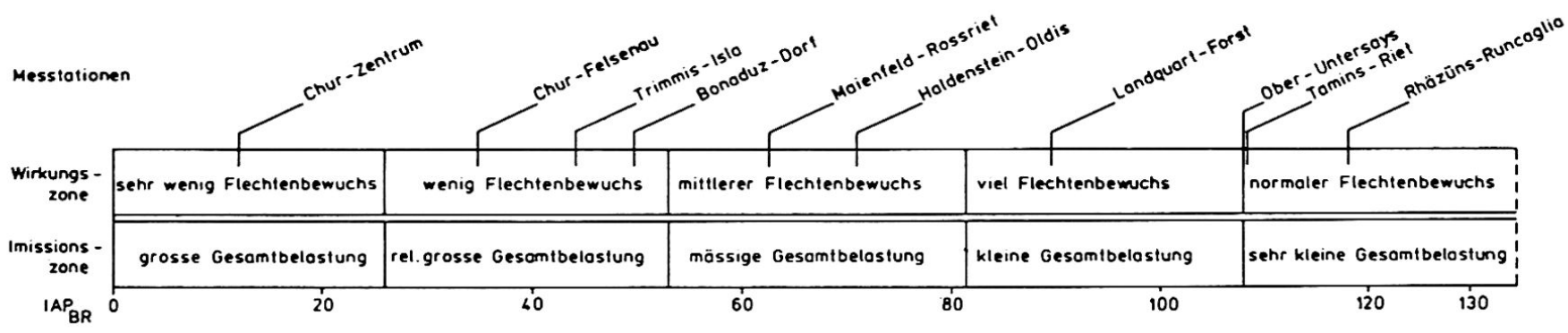

Abb. 7 Klassifizierung der Meßstationen

Flechten können als Frühwarner und zum Bestimmen der Vorbelastung eingesetzt werden. Bioindikation ermöglicht die Überprüfung der lufthygienischen Situation vor und nach Sanierungsmaßnahmen sowie bei UVP-pflichtigen Vorhaben.

Die Bioindikation mit Flechten liefert der ökologischen Planung, wie auch der Sachplanung, wertvolle Unterlagen über die Luftbelastung und deren Gefahren.

\section{Zusammenfassung}

Für das Schweizer Mittelland liegt eine Bioindikationsmethode mit Flechten vor, die gesicherte und quantitative Aussagen über die Gesamtbelastung der Luft zuläßt (HERZIG. LIEBENDÖRFER. URECH 1987). Diese Methode stößt da an Grenzen, wo sich die für das Schweizer Mittelland typische, epiphytische (baumbewohnende) Flechtenvegetation aus klimatischen Gründen ändert. Die vorliegende Untersuchung prüft die Übertragbarkeit der «kalibrierten Flechtenmethode zur Luftgütebewertung» auf Gebiete mit unterschiedlicher Flechtenflora am Beispiel des Bündner Rheintales. Die Anpassung der Flechtenmethode an andere klimatische Verhältnisse bedingte verschiedene methodische Änderungen: Die Trägerbaum-sowie Flechtenarten wurden neu ausgewählt, die Neukalibrierung der Flechtenmethode und die Beschreibung der für das Bündner Rheintal gültigen Flechtenzonen drängten sich auf.

Die «Bündner Rheintaler Flechtenmethode» liefert ebenfalls gesicherte, quantitative Aussagen über die Gesamt-Immissionsbelastung der Luft. Anhand dieser Methode wurde für das Bündner Rheintal eine fünfstufige, flächendeckende Luft-Gesamtbelastungskarte erstellt.

\section{Literatur}

FLEPP, L., SCHMID, W. A., TRACHSLER, H. (1984): Forschungsprojekt "Grundlagen und Möglichkeiten ökologischer Planung": Fallstudie Bündner Rheintal. BVR-Informationen 8, H. 2, S. 28-35

GFELLER, M., KIAS, U., SCHILTER, R., SCHMID, W. A., TRACHSLER, H., PETER, K., THOENI, L. (1987): Ökologische Planung: Fallstudie Bündner Rheintal. In: Die Umweltverträglichkeitsprüfung UVP: Publikationen der Schweizerischen Naturforschenden Gesellschaft, S. 155-159

HERZIG, R., LIEBENDÖRFER, L., URECH, M. (1985): Flechten als biologische Indikatoren der Luftverschmutzung in der Schweiz (NEP 14) - Methodenentwicklung in der Region Biel-Seeland. Systematisch-Geobotanisches Institut, Universität Bern

HERZIG, R., LIEBENDÖRFER, L., URECH, M. (1987): Flechten als Bioindikatoren der Luftverschmutzung in der Schweiz: Methoden-Evaluation und Eichung mit wichtigen Luftschadstoffen. VDI Berichte Nr. 609, S. 619-639

KIRSCHBAUM, U. (1986): Flechten als Bioindikatoren in Ballungsgebieten. AFZ 1/2, S. 10-12

LEBLANC, F., DESLOOVER, J. (1970): Relation between industrialization and the distribution and growth of epiphytic lichens and mosses in Montreal. Can. J. Bot. 48, S. $1485-1496$

MACHER, M. (1987): Flechtenwuchszonen und die Veränderung der Luftqualität in München seit 1890. In: VDI Berichte Nr. 609, S. 641-652

NASH, T. H. (1976): Lichens as indicators of air pollution. Die Naturwissenschaften 63, S. 364-367.

TRACHSLER, H., KIAS, U. (1986): Ökologische Planung Fallbeispiel Bündner Rheintal. In: Lendi, M., Reith, W. J. Schmid, W. A. (Hrsg): Ökologische Planung im Grenzraum. Schriftenreihe des Instituts für Raumplanung und agrarische Operationen der Universität für Bodenkultur Wien, Nr. 2 S. $171-196$

URECH, M., LIEBENDÖRFER, L., HERZIG, R. (1986): Flechten als biologische Indikatoren der Luftverschmutzung in der Schweiz (NEP 14) - Methodenüberprüfung an den NABELStationen. Systematisch-Geobotanisches Institut, Universität Bern 